Non-diffusive transport of suprathermal ions by intermittent turbulent structures

This content has been downloaded from IOPscience. Please scroll down to see the full text.

2016 Plasma Phys. Control. Fusion 58014023

(http://iopscience.iop.org/0741-3335/58/1/014023)

View the table of contents for this issue, or go to the journal homepage for more

Download details:

IP Address: 128.178.125.93

This content was downloaded on 06/02/2017 at 12:18

Please note that terms and conditions apply.

You may also be interested in:

Investigation of fast ion transport in TORPEX

A. Bovet, I. Furno, A. Fasoli et al.

Basic investigations of electrostatic turbulence and its interaction with plasma and suprathermal ions in a simple magnetized toroidal plasma

A. Fasoli, F. Avino, A. Bovet et al.

Three-dimensional measurements of non-diffusive fast ion transport in TORPEX

A Bovet, I Furno, A Fasoli et al.

Blob current structures in TORPEX plasmas: experimental measurements and numerical simulations

I Furno, C Theiler, D Lançon et al.

Electrostatic instabilities, turbulence and fast ion interactions in the TORPEX device

A Fasoli, A Burckel, L Federspiel et al.

Measurements of interactions between waves and energetic ions in basic plasma experiments

W W Heidbrink, $\mathrm{H}$ Boehmer, R McWilliams et al.

Transport equation describing fractional Lévy motion of suprathermal ions in TORPEX

A. Bovet, M. Gamarino, I. Furno et al.

Blob dynamics in the TORPEX experiment: a multi-code validation

F Riva, C Colin, J Denis et al.

Statistical properties of electrostatic turbulence in toroidal magnetized plasmas

B Labit, A Diallo, A Fasoli et al. 


\title{
Non-diffusive transport of suprathermal ions by intermittent turbulent structures
}

\author{
I Furno $^{1}$, A Bovet $^{1}$, A Fasoli $^{1}$, C Gauthey $^{1}, \mathrm{~K}_{\text {Gustafson }}{ }^{1}$, P Ricci $^{1}$ \\ and $B$ Ph van Milligen ${ }^{2}$ \\ ${ }^{1}$ Ecole Polytechnique Fédérale de Lausanne (EPFL), Swiss Plasma Center (SPC) CH-1015 Lausanne, \\ Switzerland \\ 2 CIEMAT-Laboratorio Nacional de Fusión, Avda. Complutense 40, 28040 Madrid, Spain \\ E-mail: ivo.furno@epfl.ch
}

Received 22 June 2015, revised 30 September 2015

Accepted for publication 6 October 2015

Published 9 November 2015

\begin{abstract}
We report on recent experimental, numerical and theoretical investigations of suprathermal ion transport and turbulent plasma dynamics in TORPEX. TORPEX is a toroidal device in which field-aligned blobs are intermittently generated and propagate across a confining magnetic field. Suprathermal ions are locally injected using a miniaturized source and detected using gridded energy analyzers. We show evidence for subdiffusive and superdiffusive transport of suprathermal ions, using an unprecedented combination of uniquely resolved three-dimensional measurements and first-principles numerical simulation. We present time-resolved measurements of suprathermal ion current fluctuations, which exhibit a clear causality relation with blobs, as revealed by a transfer entropy method for the superdiffusive case. This link is further investigated by using a conditional sampling technique, which allows resolving the cross-field dynamics of both blobs and the suprathermal ion beam, revealing that suprathermal ions experiencing superdiffusive transport are associated with bursty displacement events, resulting in highly intermittent time traces. This work links observations usually inaccessible in fusion devices and astrophysical plasmas, namely energy resolved three-dimensional time-averaged measurements, with Eulerian time-resolved measurements, which are often the only accessible measurements in such systems.
\end{abstract}

Keywords: TORPEX, turbulence, blobs, suprathermal ions

\section{Introduction}

Laboratory and astrophysical plasmas are characterized by the presence of particles with energies greater than the quasi-Maxwellian background species. Their interaction with turbulent structures can influence a wide range of plasma phenomena. Reaching controlled nuclear fusion conditions in a magnetic confinement reactor requires understanding and controlling, in plasmas with typical temperatures of the order of $20-30 \mathrm{keV}$, the transport of $\alpha$ particles, born from fusion reactions at 3.5 $\mathrm{MeV}$, and of energetic ions directly injected by neutral beams and/or generated by ion cyclotron resonance heating (ICRH) and current drive (CD) [1-3]). In astrophysical and space plasmas, energetic particles can be generated by coherent or turbulent acceleration mechanisms or by shocks $([4,5])$. Their transport properties determine, for example, the intensity of solar energetic particles, which is of great concern for space weather. Common to all of these systems is the question of how plasma turbulence influences suprathermal particles.

During the last two decades, a growing number of experimental data and theoretical findings [6, 7] point towards a failure of classical Brownian diffusive transport to adequately describe the dynamics of energetic particles. The so-called non-diffusive transport has gained growing attention in many fields, including laboratory plasma physics, fusion devices [8] and space and astrophysical plasmas [9]. In the case of non-diffusive transport, the mean-square displacement of an ensemble of particles grows nonlinearly in time

$$
\left\langle\Delta x^{2}\right\rangle \propto t^{\gamma}
$$




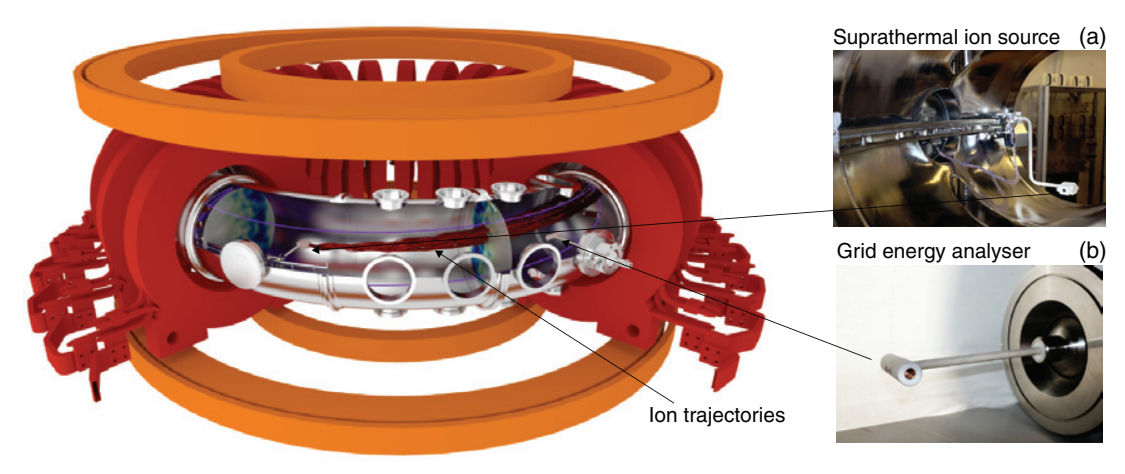

Figure 1. Experimental setup for suprathermal ion studies: view of the TORPEX vessel with magnetic field line, suprathermal ion source and detection system. Also shown are computed examples of simulated suprathermal ion trajectories with an initial energy of $30 \mathrm{eV}$. Gyromotion and the irregular spreading of the ion beam due to the interaction with the plasma turbulence is apparent. Simulated snapshots of the plasma potential are displayed at two toroidal positions.

where $\gamma \neq 1$ and $<>$ indicates ensemble-averaging. In particular, when $\gamma>1$ we have superdiffusion, and when $\gamma<1$ we have subdiffusion. The special case of classical diffusion corresponds to $\gamma=1$. In equation (1), the nonlinear growth of the mean-square displacements can be linked to the presence of non-Markovian, long memory properties in time, and/ or to nonlocal, (non-Gaussian) long range correlation properties in space $[9,10]$. In space plasmas, experimental evidence of superdiffusion of energetic particles was obtained at interplanetary shocks [11] and during solar flares [12]. Although in tokamaks, energetic particles modes, such as fishbones [13], have been observed to cause convective transport of suprathermal ions, recent experiments point towards a non-diffusive suprathermal ion transport associated with micro-turbulence [8]. Basic laboratory devices $[6,7,14]$ also suggest that the transport of suprathermal ions is generally non-diffusive.

We present recent advances in the TORPEX device [15, 16] in the understanding of non-diffusive radial transport of suprathermal ions resulting from the interaction with intermittent blobs. These are field-aligned isolated structures, where density and temperature are increased with respect to the surrounding plasma, similar to those observed at the edge and scrape-off layers of tokamaks and reversed-field pinches [17] and in the Earth ionosphere [18]. In TORPEX, blobs originate from ideal interchange waves and propagate radially outward due to $\nabla B$ and curvature induced drifts. Direct measurements of suprathermal ion transport are limited by the harsh plasma environment in fusion-grade devices and by the difficulty to perform measurements in distant astrophysical plasmas. In addition, detailed knowledge of the turbulence characteristics and of the background plasmas, necessary to realistically model the transport of suprathermal ions, have been achieved in the past years on TORPEX, which allows for easy diagnostic access and fully modelled plasma scenarios.

In section 2, the TORPEX device and the experimental setup are presented, with focus on the suprathermal ion source and detectors. In section 3, three-dimensional (3D) time-averaged measurements of suprathermal ion beam are discussed. Evidence for two transport regimes, namely subdiffusive and superdiffusive regime, is presented. In section 4, we introduce time-resolved measurements of the ion current and discuss the statistical properties of their fluctuations. A link between radially propagating blobs and the suprathermal ion transport is made in section 5 by using a statistical analysis based on the transfer entropy. Section 6 links the time-resolved measurements with the 2D dynamics of blobs in TORPEX plasmas and their effects on the suprathermal ion beam. Finally, conclusions are summarized in section 7, where the importance of the present results for fusion plasmas as well as for astrophysical and space plasmas is also discussed.

\section{Experimental setup, plasma scenario and suprathermal ion source and detectors}

TORPEX is a toroidal device with a major radius $R=1 \mathrm{~m}$ and a minor radius $a=0.2 \mathrm{~m}$, in which plasmas of different gases are produced and sustained by injecting microwaves at $2.45 \mathrm{GHz}$ in the electron cyclotron (EC) frequency range. A schematic of the device with the main elements is shown in figure 1. A flexible set of external and internal coils allows a variety of magnetic configurations, ranging from simple magnetized torus (SMT) with open magnetic field lines to closed field line configurations, and even more complex magnetic geometries, such as X-points or magnetic snowflakes [19]. For the present experiments, SMT configurations are created by combining a dominant toroidal magnetic field, $B_{\mathrm{t}} \simeq 75 \mathrm{mT}$ with a much weaker vertical magnetic field $B_{\mathrm{v}} \simeq 2 \mathrm{mT}$, resulting in a vertical magnetic field line return distance $\Delta=2 \pi R\left(B_{\mathrm{v}} / B_{\mathrm{t}}\right) \approx 18.8 \mathrm{~cm}$. An example of open magnetic field line is shown in violet in figure 1 on the left. A small level of microwave power $(\approx 400 \mathrm{~W})$ on the high field side of the TORPEX device is used to produce and sustain hydrogen plasmas. Typical electron temperature and density are $T_{e} \simeq 1-6 \mathrm{eV}, n_{e} \simeq 10^{15}-10^{16} \mathrm{~m}^{-3}$ (see $[14,20,21]$ for the radial profiles of the relevant plasma parameters), while the ions are cold $T_{i}<1 \mathrm{eV}$. As revealed by comprehensive experimental investigations [22-31] and 3D global fluid simulations [32-34], these plasmas are characterized by the presence of an ideal interchange mode, localized around the region of maximum pressure gradient (with a perpendicular wave number 
$k_{\Delta} \simeq 35 \mathrm{rad} \cdot \mathrm{m}^{-1}$, and a wave number parallel to the magnetic field, $k_{\|} \simeq 0$ ). This ideal interchange mode is driven by the magnetic curvature and the pressure gradient and intermittently generates field-aligned blobs, which propagate radially outward resulting in intermittent transport of particles, heat, and momentum $[35,36]$ and exhibit universal statistical properties $[37,38]$ similar to those observed in tokamaks. In these fully characterized plasmas, suprathermal lithium $6^{+}$ions are injected in the blob region using a miniaturized ion source and detected using gridded energy analyzers (GEA), which were specifically developed for these experiments. These are described in details below.

A dedicated miniaturized $\mathrm{Li}^{+}$ion source (figure 1(a)) was developed in collaboration with the University of California at Irvine, based on the design of the orignal source used to perform similar experiments on LAPD [39]. The source consists of a Li-doped thermionic aluminum-silicate emitter producing $\mathrm{Li6}^{+}$ion currents up to $10 \mu \mathrm{A}$ [40]. The emitter is encased into a vacuum and plasma safe boron nitride casing, which includes a two-grid system to accelerate the ions. A screen grid is grounded to minimize perturbations, while the voltage between the emitter and the accelerating grid is modulated. The accelerating grid is powered by a high voltage DC-power supply. $\mathrm{Li}^{+}$ions with energies in the range $20 \mathrm{eV}-1 \mathrm{keV}$ can be produced. The suprathermal ion source is mounted on a moving system, as shown in figure 1(a), and can be positioned at different locations over a toroidal distance of about $2 \mathrm{~m}$ in between discharges.

Ion energy and current density profiles are measured using two identical GEAs, figure 1(b), which face opposite directions for background noise subtraction. Each detector has small dimensions relative to the plasma size $(15 \mathrm{~mm}$ in diameter, $70 \mathrm{~mm}$ in length and in inlet diameter of $8 \mathrm{~mm}$ ), and is able to measure fast ion currents as small as $0.1 \mu \mathrm{A}$. The GEA detector is installed on a 2D (radial-poloidal movement) positioning system as shown in figure 1(b), which allows reconstructing the ion current density profile with a spatial resolution of $2 \mathrm{~mm}$ over almost the entire poloidal cross section by adapting the distance between measurements points to the profile shape.

This ion source and GEAs setup allow measurements of the $3 \mathrm{D}$ properties of the suprathermal ion beam as it interacts with blobs. For the present measurements, two detection schemes can be used: (1) synchronous detection by modulating the emitter bias voltage at a given frequency $(\approx 1 \mathrm{kHz})$ to improve the signal-to-noise ratio [41], which allows measuring time-averaged profiles (section 3); (2) time-resolved measurements allowing for the evaluation of the statistical properties of the suprathermal ion current fluctuations (sections 4 and 5) and the temporal dynamics of the suprathermal ion beam (section 6) .

\section{Super- and sub-diffusive regimes for suprathermal ion transport}

This section reviews the experimental measurements of timeaveraged, 3D spatial profiles of suprathermal ions obtained on TORPEX in the past few years [14, 20, 21]. An unprecedented combination of experiments, first-principles numerical simulations using the GBS fluid code [34], and theory understanding allowed the identification of two transport regimes for the suprathermal ions, namely subdiffusive and superdiffusive regimes. This provides the background for the timeresolved measurements of the following sections.

The experiments are performed by injecting suprathermal ions in the toroidal direction at two different energies, $30 \mathrm{eV}$ and $70 \mathrm{eV}$, in the scenario described in section 2. The Larmor radius for $70 \mathrm{eV}$ and $30 \mathrm{eV}$ ions is $\sim 0.8 \mathrm{~cm}$ and $\sim 0.5 \mathrm{~cm}$, respectively. The ion source is positioned at $x=1.5$ and $y=-4.3 \mathrm{~cm}$ in the blob region, where the electron temperature is $\approx 1.3 \mathrm{eV}$. Time-averaged poloidal profiles of the suprathermal ion current at different toroidal locations are shown in figure 2 for two different energies, $E=70 \mathrm{eV}$ ((a), (b)) and $E=30 \mathrm{eV}((\mathrm{c}),(\mathrm{d}))$. The spreading of the suprathermal ion beam is quantified by the radial variance of the $2 \mathrm{D}$ poloidal profile as a function of the toroidal distance in figure 2(e). In the absence of a plasma, the ion motion in the SMT configuration results from the combination of gyromotion and $\nabla B$ and curvature-associated vertical drift of the gyrocenters. This latter is clearly evident in the profiles in figures 2(a)-(d), where the oscillations of the beam witdh in figure 2(e) result from the gyromotion of the ions, which are injected with a small spreading in their Larmor radii. Figure 2 shows that, on top of the unperturbed motion observed in the absence of a plasma, a broadening due to the interaction with the plasma turbulence is present, together with a convective transport associated with the background $\mathbf{E} \times \mathbf{B}$ velocity field. This latter results in a vertical displacement of the suprathermal ion beam and not in radial transport. The beam spreading is different for the two suprathermal ion energies. While close to the ion source, the two profiles have similar radial widths, as the distance from the source is increased, the broadening of the $30 \mathrm{eV}$ beam is larger than that of the $70 \mathrm{eV}$ ions. This suggests that different turbulent transport regimes may be at play for the two ion energies. We note that collisions between suprathermal Li ions and neutral hydrogen can be neglected, in the present experiments. For a neutral gas pressure of $5 \times 10^{-5}$ mbar, the collision frequency for collisions between suprathermal Li ions and neutral hydrogen is approximately $6.2 \mathrm{kHz}$, which results in a mean free path between collisions of $\approx 8 \mathrm{~m}$. This is much longer than the distance travelled by the suprathermal ions between the source and the detection point, resulting in a negligible contribution of ion-neutral collisions to the suprathermal ion transport.

Although 3D suprathermal ion beam profiles are directly measured in TORPEX, this is not sufficient to identify the transport regime, which requires evaluating the radial transport exponent, $\gamma_{R}$. This must be computed using equation (1) from the temporal dependence of the variance of the displacements of the suprathermal ions, which is clearly not accessible from the experimental data of figure 2. Here, we use numerical simulations $[42,43]$ reproducing the experimental measurements and compute directly $\gamma_{R}$. A large number of tracers $\left(1.6 \times 10^{5}\right)$, mimicking the source experimental setup, are injected in global fluid turbulent simulations that were 

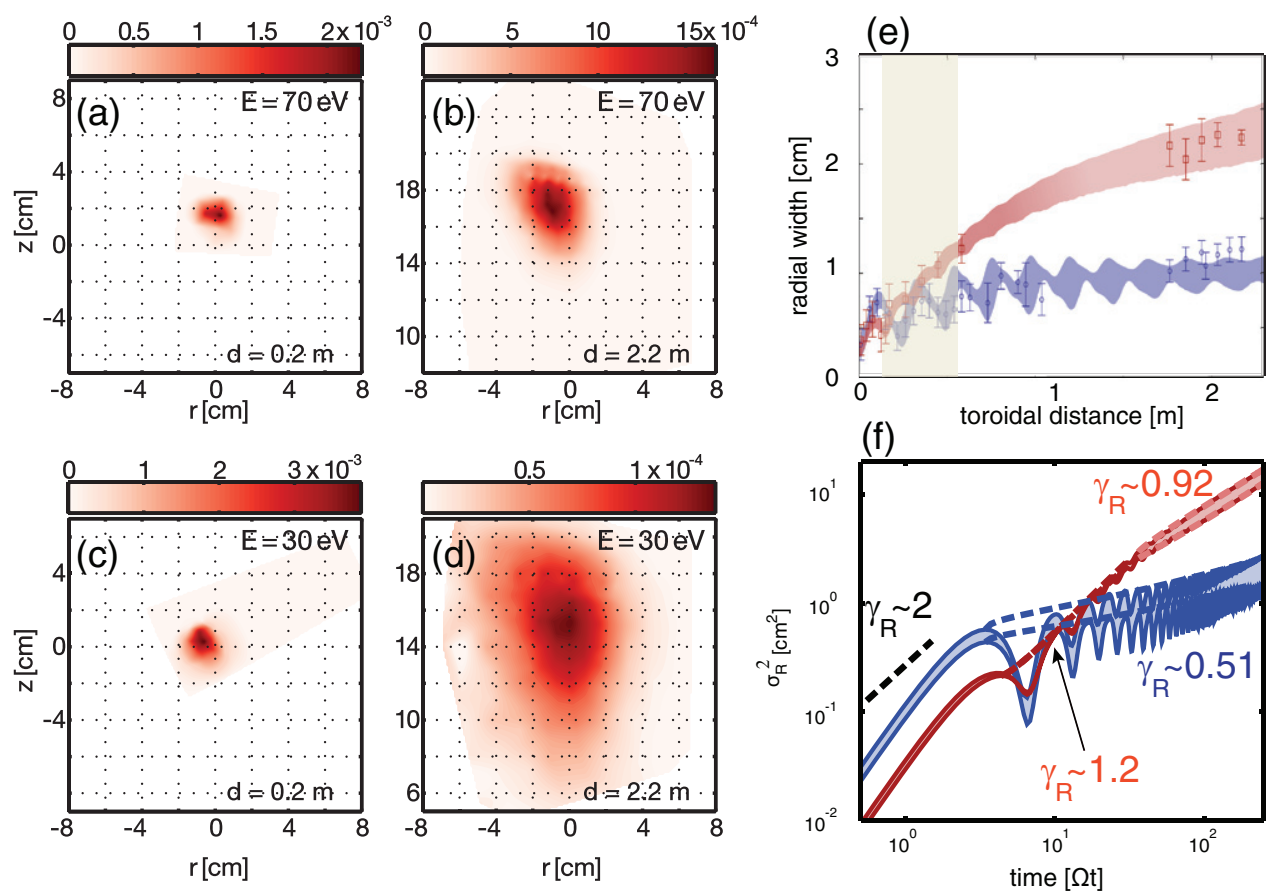

Figure 2. On the left: poloidal profiles of suprathermal ion current at different toroidal distances ((a), (b)) for $70 \mathrm{eV}$ and ((c), (d)) $30 \mathrm{eV}$ ions. (e) Dependence upon the radial distance from the source of the radial width of the suprathermal ion current profiles. Blue dots and red squares represent the experimental measurements for the $70 \mathrm{eV}$ and and $30 \mathrm{eV}$ ions respectively. The continuous bands are obtained from numerical simulations of the using a synthetic diagnostics. The shaded grey area approximately identifies the region where $30 \mathrm{eV}$ and $70 \mathrm{eV}$ ions are superdiffusive and subdiffusive, respectively. (f) Variance of the radial positions of the suprathermal ions as a function of time from the numerical simulations in (e). Power-law fits in the different phases, shown by the dashed lines, provide the values of the transport exponent $\gamma_{R}$.

previously validated against experimental data. The tracer trajectories are computed using the Newton equation of motion. The results of the simulations are compared with the experimental measurements with the help of a synthetic diagnostic reproducing the suprathermal ion current profile measured with the detectors. This procedure is illustrated in figure 2(e), which shows two simulations reproducing within experimental uncertainties the present experiments.

Using these simulation results, the temporal evolution of $\sigma_{R}^{2}$ is readily accessible, as shown in figure 2(f). This allows computing the value of the radial transport exponent, $\gamma_{R}$ by fitting its temporal evolution to power laws in different phases. For the two ion energies, we note that at first the transport is ballistic $\left(\sigma_{R}^{2}(t) \propto t^{2}\right)$, which corresponds to a phase during which the ions have not yet interacted with the plasma and are not yet magnetized. The ions enter a second spreading phase (corresponding approximately to the shaded area in figure 2(e)) as they start to interact with the plasma turbulence, where different transport regimes are observed, depending on the energy of the ions. In this second phase, the $30 \mathrm{eV}$ ions experience a superdiffusive transport corresponding to $\gamma_{R} \approx 1.2$ while the transport of the $70 \mathrm{eV}$ ions is subdiffusive with $\gamma_{R} \approx 0.51$. A numerical study shows that in this interaction phase the transport can vary from a subdiffusive to superdiffusive regime depending on two parameters that determine the relative sizes of the ion orbits and the turbulent structures: the injection energy normalized to the electron temperature, $E / T_{e}$, and the normalized fluctuations amplitude, $e \tilde{\phi} / T_{e}$.
The late evolution of the ions is also different for the two energies: while the $30 \mathrm{eV}$ ions experience a quasi-diffusive transport $\left(\gamma_{R} \approx 0.92\right)$, the most energetic ions remain subdiffusive. This third phase starts from a distance from the source of approximately $1 \mathrm{~m}$, or, in time, about six gyroperiods in figure 2(f), and appears when the size of the beam is sufficiently large that the ions sample region of the plasma with different fluctuation amplitude.

\section{Time-resolved measurements and statistical properties of suprathermal ion fluctuations}

In this section, we perform time-resolved measurements of the suprathermal ion current in the poloidal cross section at a distance $d=41.2 \mathrm{~cm}$ from the source, where different transport regimes are observed for the two ion energies. For these measurements, the lock-in system used for synchronous detection section 2 is bypassed, thus allowing the GEAs to directly acquire the time evolution of the suprathermal ion current [44]. To discriminate between background noise and suprathermal ion current fluctuations, the source is modulated at $\approx 30 \mathrm{~Hz}$.

Two examples of ion current time-traces are shown in figures 3(a) and (b) for energies of $30 \mathrm{eV}$ and $70 \mathrm{eV}$, respectively. The position of the measurements in the poloidal plane is indicated by a cross in figures 3(c) and (d). The ON-phase and OFF-phase of the ion source are shown in red and blue respectively. We note that, during the ON-phase, the time trace for 

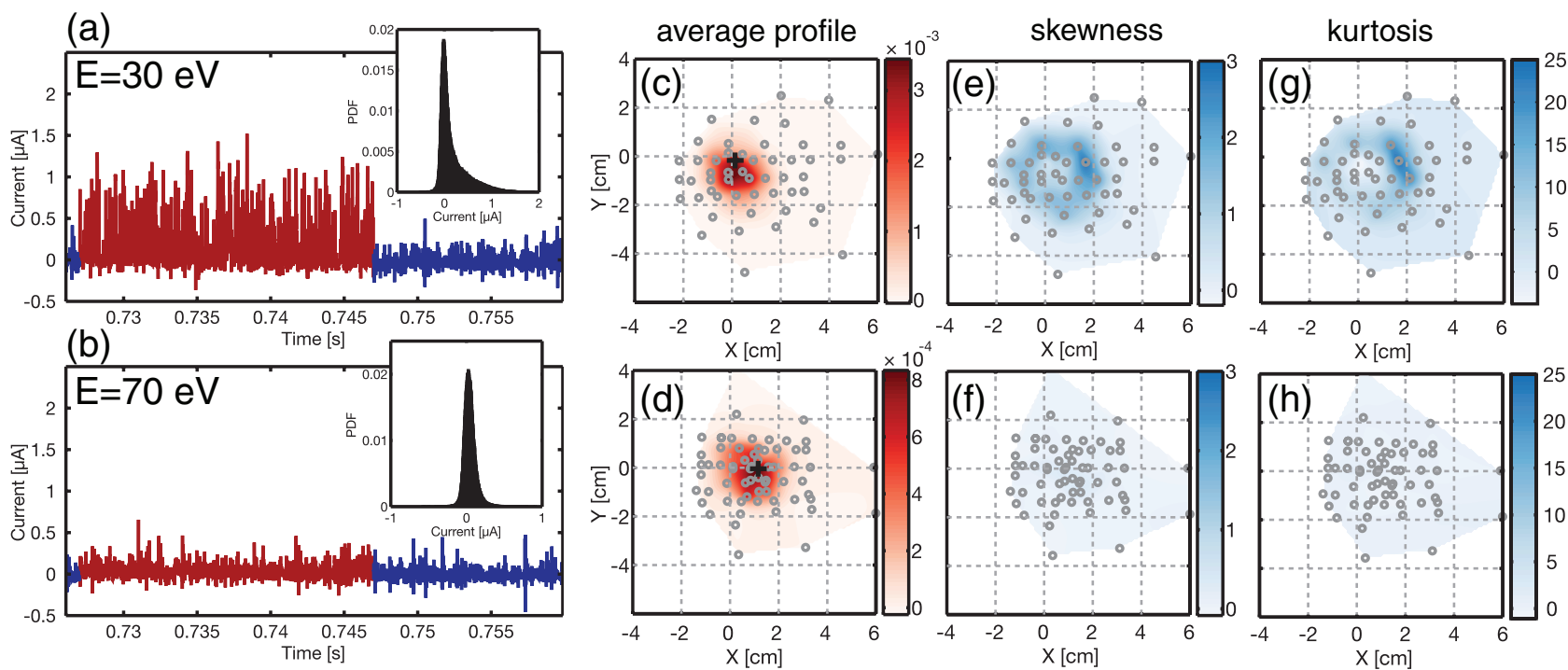

Figure 3. ((a), (b)) Ion Current density traces measured by the GEA at the location indicated by the black cross and $42.1 \mathrm{~cm}$ away from the source. The ion source is turned ON (red) and OFF (blue) at $30 \mathrm{~Hz}$ frequency to discriminate the background noise. The insets show the PDFs of the ion current fluctuations. Poloidal profiles of ((c), (d)) time-averaged suprathermal ion current density in A m ${ }^{-2}$, ((e), (f)) skewness and $((\mathrm{g}),(\mathrm{h}))$ kurtosis of the ion current density fluctuations.

the $30 \mathrm{eV}$ ions, experiencing superdiffusive transport, exhibits a high level of intermittency, characterized by the presence of large bursts of positive amplitude. Conversely, for the $70 \mathrm{eV}$ ions, which are in the subdiffusive regime, the current time trace is not strongly intermittent and does not show large amplitude fluctuations. To characterize the intermittency of the time traces, we compute the skewness $S$ and kurtosis $K$ of their probability distribution functions (PDFs). The skewness is the normalized third order moment of the PDF and measures its asymmetry. The kurtosis is the normalized fourth order moment of the PDF and is a measure of the heaviness of the PDF tails. The PDFs for the ion current traces are shown in the insets in figures 3(a) and (b). The PDF for the $30 \mathrm{eV}$ ions is positively skewed, as demonstrated by the asymmetry towards large amplitude bursty events, while the PDF for the $70 \mathrm{eV}$ ions is almost Gaussian.

Figures 3(a)-(g) displays 2D poloidal profiles of the PDF moments of the suprathermal ions current density for the two energies. The OFF-periods are used to compute the positiondependent background values of the mean (offset), skewness and kurtosis, which are then removed from the values computed during the ON-phase. Although the time-averaged profiles for the two energies (figures 3(c) and (d)) are relatively similar, when the profiles of the skewness and kurtosis are reconstructed a clear difference appears. The skewness and kurtosis profiles for the $70 \mathrm{eV}$ ion beam are flat, while the profiles for the $30 \mathrm{eV}$ ions reveal a region of high skewness and high kurtosis around the peak of maximum time-averaged current. This implies that, in the peripheral regions of the $30 \mathrm{eV}$ profile, where the time traces have a low time-averaged current compared to the center of the profile, the intermittency is more important. These observations provide evidence for a clear difference in the statistical properties of suprathermal ion current fluctuations, for ions experiencing different transport regimes. At the same time, the strong intermittence observed in the superdiffusive regimes is reminiscent of the intermittence associated with the generation/propagation of blobs in the scenarios under investigations and suggests that superdiffusion may be linked to the interaction of suprathermal ions with blobs.

\section{Entropy transfer between ion-saturation current signals and suprathermal ion current}

To investigate the relation between the suprathermal ion transport and the background plasma turbulence, we use a method for causality detection, that was originally developed in the field of information theory [45], based on the transfer entropy. This method has been recently applied in plasma physics to detect causality links between turbulent fluxes and zonal flows [46] and is briefly reviewed below.

Consider two discretely sampled, stationary time series data $x_{i}$ and $y_{j}$, generated by two processes $X$ and $Y$. A measure of the transfer of information between $X$ and $Y$ is given by the transfer entropy

$$
T_{Y \rightarrow X}=\sum p\left(x_{n+1}, x_{n}^{(k)}, y_{n}^{(l)}\right) \log _{2} \frac{p\left(x_{n+1}, x_{n}^{(k)}, y_{n}^{(l)}\right) p\left(x_{n}^{(k)}\right)}{p\left(x_{n}^{(k)}, y_{n}^{(l)}\right) p\left(x_{n+1}, x_{n}^{(k)}\right)}
$$

where $p$ is a joint probability distribution function, the sum runs over its arguments, and $x_{n}^{(k)}=\left(x_{n-\alpha_{k}}, \ldots, x_{\alpha_{1}}\right)$ and $y_{n}^{(l)}=\left(y_{n-\beta_{l}}, \ldots, y_{\beta_{1}}\right)$ indicate a set of $k, l$ data values preceding or coinciding with the time associated with the time index $n$. These values are identified by the multi-indices $\alpha=\left(\alpha_{1}, \alpha_{2}, \ldots, \alpha_{k}\right)$ and $\beta=\left(\beta_{1}, \beta_{2}, \ldots, \beta_{k}\right)$. If $Y$ has no influence on the future evolution of the variable $X$, i.e. it is not causally linked to it, then $T_{Y \rightarrow X}=0$. Note that the evaluation of equation (2) is performed by discrete binning the PDFs with $m$ bins in each dimension, such that it requires the computation of four multi-dimensional PDFs, which is numerically demanding and time consuming. For this reason, usually 

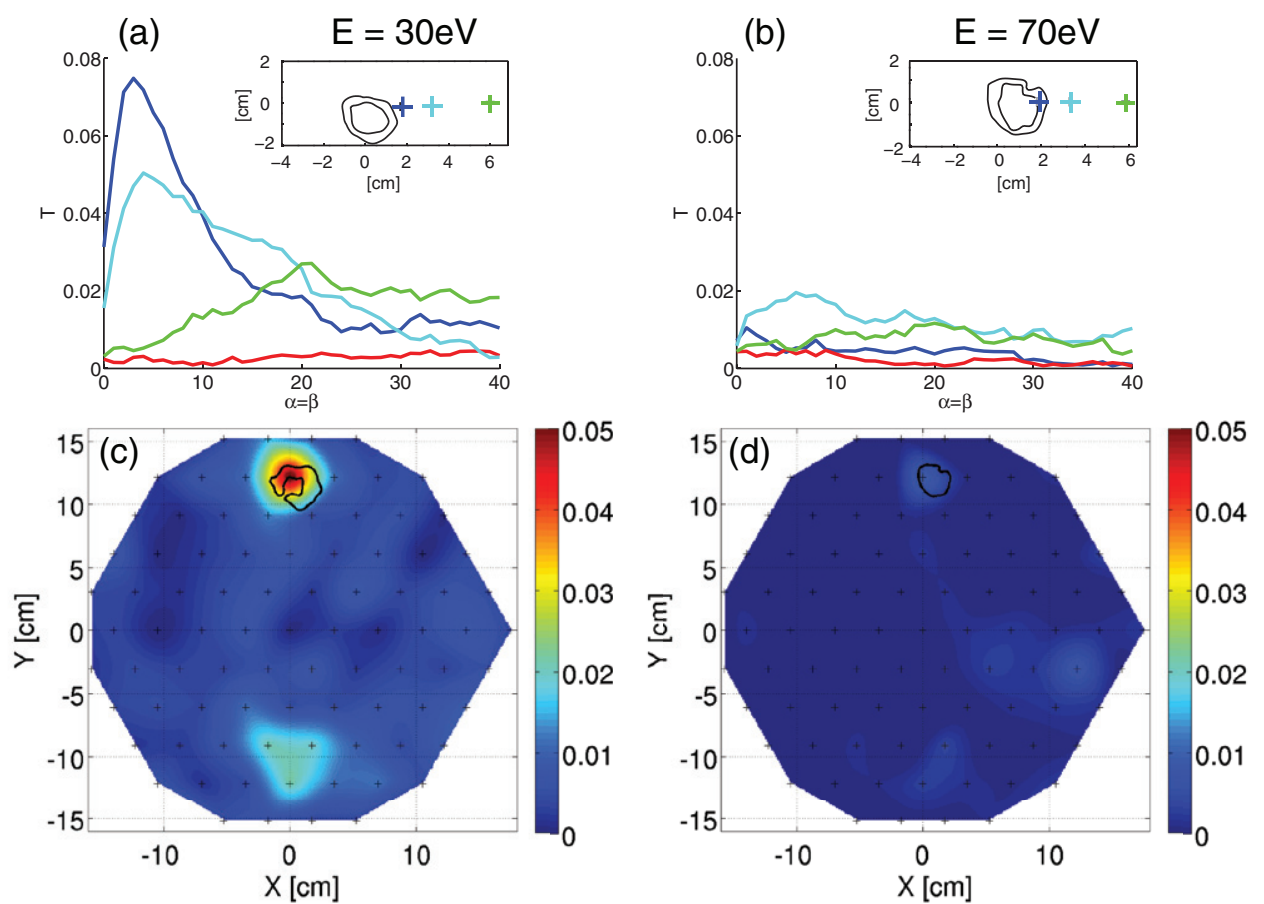

Figure 4. ((a), (b)) For the two ion energies, transfer entropy computed using equation (2) between the HEXTIP tip at $X=0$ and $Y=12 \mathrm{~cm}$ and the GEA signals recorded at the positions indicated by the crosses (same colors) in ((c), (d)). For the same signals providing the blue curve, the red curve shows the reverse transfer entropy, indicating that the causality link is negligible in the backward direction. ((c), (d)) Poloidal profiles of the mean Entropy Transfer $\langle T\rangle$ (averaged over the entire suprathermal ion beam signals) indicating the position of the maximum forward interaction.

the choice $l=k=1$ is made together with coarse-graining of the PDFs, corresponding to $m=3$.

With the main objective to analyze the interaction between blobs and suprathermal ions, we compute the transfer entropy in equation (2) using ion saturation current time series from the HEXTIP array of Langmuir probes (see below) and the time-resolved measurements of the suprathermal ion current discussed in section 3. The HEXagonal turbulence imaging probe (HEXTIP) is an array of 85 LPs at one toroidal location covering the entire poloidal cross section with a spatial resolution of $3.5 \mathrm{~cm}$. HEXTIP allows characterizing fluctuations and turbulent structures in terms of wavelength, coherence lengths, frequency, speed and statistical properties, in a single plasma discharge ([47]) and constitutes the workhorse for a high temporal resolution $(250 \mathrm{kHz})$ real space imaging of the ion saturation current and floating potential dynamical evolution. For the following analysis only $I_{\text {sat }}$ signals are considered and this will allow in the next section to link the transfer entropy analysis with 2D blob dynamics.

In figures 4(a) and (b), we show the transfer entropy

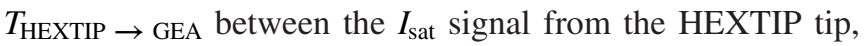
located at $X=0$ and $Y=12 \mathrm{~cm}$ and three suprathermal ion current signals from the GEA location indicated by crosses in the insets in figures 4(a) and (b) for the $30 \mathrm{eV}$ and $70 \mathrm{eV}$ case. $T_{\text {HEXTIP } \rightarrow \text { GEA }}$ is as a function of the parameters $\alpha=\beta$, which corresponds to time indices ( $\Delta t=4 \mu \mathrm{s}$ ). In the same figure, as an example, we also show in red one reversed transfer entropy $T_{\mathrm{GEA}} \rightarrow$ HEXTIP, for one single GEA tip. This is much smaller than the corresponding forward transfer entropy (in blue in figures 4(a) and (b)) and reveals a causal relation between the ion saturation turbulent fluctuations and not viceversa, confirming the tracer character of the injected ions. For the $30 \mathrm{eV}$ case in figure 4(a), the causality relation is clearly revealed by the large value of the Transfer entropy, which peaks around $20 \mu \mathrm{s}$ (corresponding to $\alpha=\beta=4$ ). Signals corresponding to GEA positions further from the suprathermal ion beam center display a smaller level of causality dependence, which also peaks at later time. We should also note that the transfer entropy is much larger in the case of the $30 \mathrm{eV}$ ions than for the $70 \mathrm{eV}$ case. This indicates that the plasma turbulence, quantified here by $I_{\text {sat }}$ fluctuations, and therefore radially propagating blobs are interacting more strongly with the $30 \mathrm{eV}$ beam than the $70 \mathrm{eV}$ beam. This explains the observed difference in the statistical properties of of the suprathermal ion current fluctuations discussed in section 4 .

The full spatial coverage of the HEXTIP array allows identifying the region of the plasma that is more strongly interacting with the suprathermal ion beam. For this, we compute the maximum transfer entropy $T_{\mathrm{HEXTIP}, j} \rightarrow$ GEA,$i$ between the HEXTIP tip $j$ (we choose the tip that interacts the most strongly) and GEA at the position indicated by $i$. Figures 4(c) and (d) presents the poloidal distribution of $T$ for both energies, clearly revealing that most of the interaction happens for the $30 \mathrm{eV}$ case with turbulence that is localized (within experimental uncertainties) at approximately the same position of the beam. In the lower half of the poloidal cross section figure 4(c), a second maximum of $T$ is clearly visible, which is due to the field aligned nature of the turbulent blobs in this scenario and the fact that the magnetic field lines perform approximately two toroidal turns before reaching the vessel walls. 

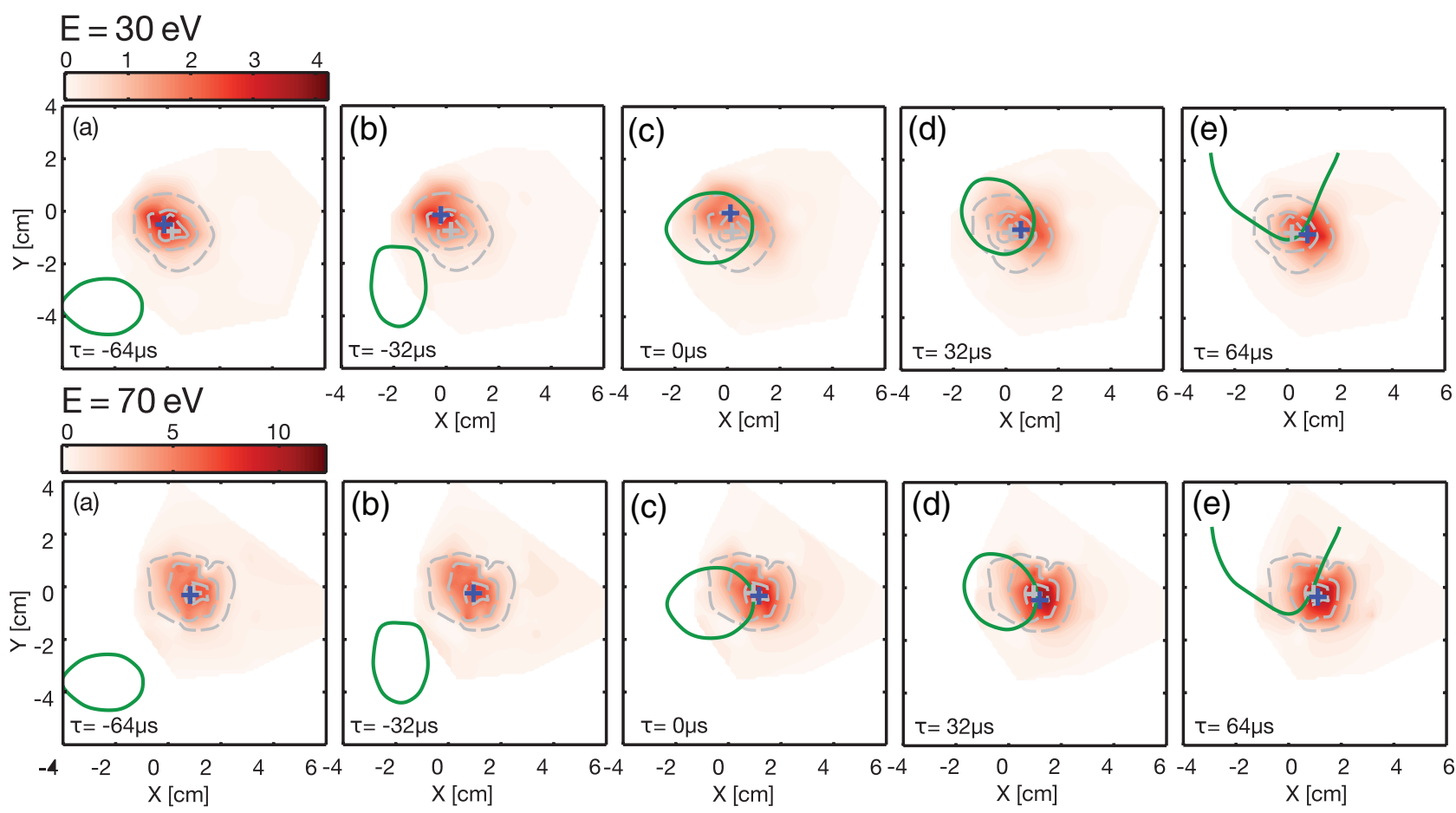

Figure 5. Conditionally averaged dynamics of the suprathermal ion current density $\left(\mathrm{A} \mathrm{m}^{-2}\right)$ for the two energies. Dashed gray contours represent time avergaed profiles and the green lines represent the CAS blob $I_{\text {sat }}$ iso-contours. The center of mass positions of the beam at different times is indicated by the blue cross.

\section{Time-resolved conditionally sampled spatial dynamics of suprathermal ion transport}

The interaction between blobs and suprathermal ions is further investigated using a conditional averaging sampling (CAS) technique that allows accessing the time-resolved dynamics of the suprathermal ion beam. The CAS technique is described in details in [48]. Using an ion saturation $I_{\text {sat }}$ reference signal from a HEXTIP probe, we detect events defined as bursts in $I_{\text {sat. }}$ These bursts are characterized by $I_{\text {sat }}>3 \sigma$, where $I_{\text {sat }}$ is the ion saturation current fluctuations, and $\sigma$ is the standard deviation of the ion saturation current signal. The suprathermal ion current is averaged in a time window centered around each event. This procedure is applied to the phases with and without suprathermal ions. The difference between the two CAS shows the dynamics of the suprathermal ion beam when an event is detected on a given probe tip of HEXTIP. We note that since HEXTIP provides a full coverage of the poloidal cross section, the CAS technique applied with the same reference probe provides the spatio-temporal evolution of propagating blobs, which are responsible for the intermittency of the HEXTIP signals.

The results of the CAS technique are summarized in figure 5, which shows the time evolution of the conditionallyaveraged suprathermal ion current profile for the two energies. The reference probe is located at $X=0$ and $Y=12.2 \mathrm{~cm}$ and corresponds to the probe that has a maximum entropy transfer as shown in figure 4(c). Due to the helical magnetic configuration, blobs detected on this tip are aligned with a magnetic field line that is located at $X=0$ and $Y=-0.5 \mathrm{~cm}$ at the toroidal location of the detector, which is close to the maximum of the time-averaged profiles. This is displayed by the dashed gray lines and its maximum is indicated by the gray cross. The CAS analysis is performed from $64 \mu$ s before to $64 \mu \mathrm{s}$ after the detection of the blob. This time interval corresponds approximately to the autocorrelation time of floating potential fluctuations in the blob region and of $I_{\text {sat }}$ fluctuations. In the same figure, we show the dynamics of the CAS blob using a density iso-contour (green line). We note that the blob dynamics is almost exactly identical for the two suprathermal ion energies, since the ions are injected in similar plasmas. This confirms that they can be considered as tracers (i.e. they do not back react on the plasma itself).

For the $30 \mathrm{eV}$ case (top row in figure 5), the beam is first displaced to the top-left with respect to its time-averaged profile (gray contours) ((a), (b)). Then, when the blob crosses the probe ((c)-(e)), the displacement changes from left to right. At later times ((g)-(i)), the beam is displaced to the bottom right of its averaged position. The time scale of the beam displacement is comparable to the duration of the intermittent bursts shown in figure 3(a). Figure 5 at the bottow row shows that as the same CAS blob crosses the $70 \mathrm{eV}$ ion beam, the displacement of the beam is extremely small compared to that of the $30 \mathrm{eV}$ beam. This indicates that the background intermittent turbulence has very little effect on the $70 \mathrm{eV}$ suprathermal ion beam, which is consistent with a small value of the transfer entropy, as found in the previous section.

The mechanisms perturbing the trajectories of suprathermal ions is illustrated in figure 6(a), which shows the effect of blobs at different positions in the poloidal plane. We use as reference probe HEXTIP tips at different vertical positions between the radial position $0 \mathrm{~cm} \leqslant X \leqslant 1.7 \mathrm{~cm}$. From the 

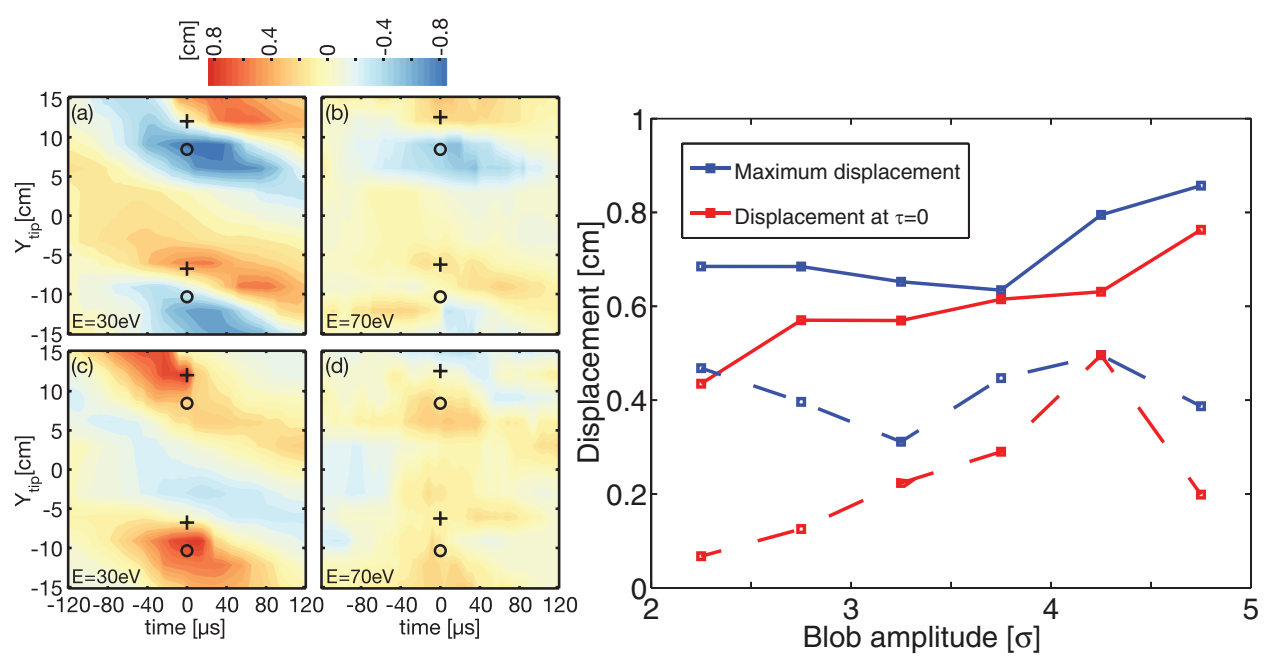

Figure 6. Left: Horizontal (top row) and vertical (bottom row) displacement of the suprathermal ion beam due to large blobs. On all panels, the $x$-axis represents the time relative to the detection of the blob on the reference probe and the $y$-axis represents the vertical position of the reference probe. The crosses and the circles indicate the positions of the beam center, when it reaches the detector, and of the source, respectively, projected along the helical magnetic field lines on the poloidal plane of the reference probes. Right: maximum displacement (blue) and displacement at $\tau=0$ of the ion beam for the $30 \mathrm{eV}$ (continuous lines) and $70 \mathrm{eV}$ (dashed lines) case as a function of the blob amplitude. This latter is measured in units of standard deviation. Error bars of the beam position measurements are $\pm 1 \mathrm{~mm}$. These are computed by modeling the measurement with a finite size detector on analytic profiles (2D Gaussians with the same size than the experimental profiles) using a constant resolution of $0.4 \mathrm{~cm}$ and a normally distributed error on the detector position (with a standard deviation of $2 \mathrm{~mm}$ ). The beam position is then computed using the same method used on the experimental profiles and compared the results with the prescribed position.

CAS, we compute the horizontal and vertical displacement of the beam center of mass as a function of time. These are shown in figure 6 (a) for $30 \mathrm{eV}$ and $70 \mathrm{eV}$ ions due to large blobs, i.e. $I_{\mathrm{sat}}>4 \sigma$. The circles represent the position of the suprathermal ion source projected along the helical magnetic field lines on the poloidal plane of the reference probes. The crosses represent the position of the beam center, when it reaches the detector, projected onto the same plane. Thus, the vertical distance between the circles and the crosses show the vertical distance traveled by the ions between their emission and their detection due to the vertical drift. We observe that $30 \mathrm{eV}$ ions are systematically more significantly displaced by the blobs than $70 \mathrm{eV}$ ions and also that, depending on its position relevant to that of the blob, the beam can be displaced inwards or outwards. The beams are not displaced when a blob transits far away (for probes located at $Y \simeq 0 \mathrm{~cm}$ ). We observe that blobs passing above the beam tend to move it outwards and that blobs passing below it tend to move it inwards. The plasma potential associated with blobs in TORPEX has been identified with a bipolar structure that results from the $\nabla B$ and curvature-induced charge separation inside them [27]. The observed suprathermal ion beam displacement is consistent with the bipolar potential structure, taking into account that in the present plasma conditions the blob motion can have a strong vertical component.

To investigate the link between the blob amplitude and the displacement of the suprathermal ion beam for the $30 \mathrm{eV}$ case, we perform the conditional sampling analysis over five different classes of blob amplitudes defined by the conditions $(2+j / 2) \sigma \leqslant \widetilde{I}_{\text {ref }} \leqslant(2.5+j / 2) \sigma$ with $j=0,1,2, \ldots, 5$. For each class, the temporal blob dynamics is similar to that in figure 5 . Figure 6 shows the dependence upon the blob amplitude of maximum displacement (blue) and displacement at the time of the blob detection. While for the $70 \mathrm{eV}$ case the blob effect on the suprathermal ion beam is small, in the $30 \mathrm{eV}$ case we note that larger amplitude blobs give rise to larger beam displacements.

\section{Summary and discussion}

In summary, thanks to unprecedented time-resolved measurements, we show that suprathermal ions in TORPEX plasmas experiencing superdiffusive transport are associated with bursty displacement events resulting in highly intermittent time traces. Suprathermal ions experiencing subdiffusive transport do not display such intermittency. The intermittency of the time traces is quantified by the skewness and kurtosis of their fluctuations. For superdiffusive ions, the skewness and kurtosis poloidal profiles reveal a ring-shaped region around the peak of maximum current, where the fluctuations are the most intermittent. Using measurements, we prove that the intermittency in the superdiffusive ion case is due to their higher sensitivity to intermittent blobs, which move the ions through their electrical field either inwards or outwards depending on their relative location. Larger blobs have a greater effect than smaller blobs. From the single particle point of view, as discussed in $[6,7,14]$, the transport regime in TORPEX is ultimately linked to the effect of gyro-averaging and/or driftaveraging. In the first case, when the size of the ion orbits become comparable to characteristic dimensions of the turbulent structures, the suprathermal ions effectively average the turbulent electric field during their gyromotion thus reducing the radial transport. Gyro-averaging is also expected to be at play in fusion devices. In these latter, turbulent transport 
of suprathermal ions confined in the core plasma is due to broad-band turbulence and not directly linked to the presence of blobs, which are observed at the edge and in the scrape-off layer region. While in TORPEX the relevant size for gyroaveraging is determined by the blob size, in fusion devices for core-confined suprathermal ions, the transport regime is likely to depend upon the size of the turbulent structures generated by broad-band turbulence.

Our work links observations usually unaccessible in fusion devices and astrophysical plasmas, namely energy-resolved three-dimensional time-averaged measurements, with Eulerian time-resolved measurements, which are often the only accessible measurements in such experiments. The presence of large fluctuations in the time traces of superdiffusive ions corroborates our previous theoretical studies, which show that heavy-tailed Lévy statistics govern this regime [14, 49].

\section{Acknowledgments}

This work is partly supported by the Fonds National Suisse de la Recherche Scientifique. Fruitful discussions with members of the team 297 'Superdiffusive Transport in Space Plasmas and Its Influence on Energetic Particle Acceleration and Propagation' at the ISSI are kindly acknowledged.

\section{References}

[1] Weisen H, Furno I and TCV Team 2001 Particle transport with high power central ech and eccd in TCV Nucl. Fusion 411227

[2] Goodman T P et al 2003 An overview of results from the TCV tokamak Nucl. Fusion 431619

[3] Henderson M A et al 2003 Recent results from the electron cyclotron heated plasmas in tokamak à configuration variable (TCV) Phys. Plasmas 101796

[4] Reames D V 1999 Particle acceleration at the Sun and in the heliosphere Space Sci. Rev. 90 413-91

[5] Perri S and Zimbardo G 2012 Superdiffusive shock acceleration Astrophys. J. $\mathbf{7 5 0} 87$

[6] Gustafson K, Ricci P, Bovet A, Furno I and Fasoli A 2012 Suprathermal ion transport in simple magnetized torus configurations Phys. Plasmas 19062306

[7] Gustafson K, Ricci P, Furno I and Fasoli A 2012 Nondiffusive suprathermal ion transport in simple magnetized toroidal plasmas Phys. Rev. Lett. 108035006

[8] Heidbrink W W, Park J M, Murakami M, Petty C C, Holcomb C and Van Zeeland M A 2009 Evidence for fast-ion transport by microturbulence Phys. Rev. Lett. 103175001

[9] Perrone D et al 2013 Nonclassical transport and particle-field coupling: from laboratory plasmas to the solar wind Space Sci. Rev. 178 233-70

[10] del Castillo-Negrete D, Carreras B A and Lynch V E 2005 Nondiffusive transport in plasma turbulence: A fractional diffusion approach Phys. Rev. Lett. 94065003

[11] Perri S and Zimbardo G 2009 Ion superdiffusion at the solar wind termination shock Astrophys. J. Lett. $693 \mathrm{~L} 118$

[12] Trotta E M and Zimbardo G 2011 Quasi-ballistic and superdiffusive transport for impulsive solar particle events Astronomy Astrophys. 530 A 130

[13] White R B, Goldston R J, McGuire K, Boozer A H, Monticello D A and Park W 1983 Theory of mode-induced beam particle loss in tokamaks Phys. Fluids $\mathbf{2 6} 2958$
[14] Bovet A, Gamarino M, Furno I, Ricci P, Fasoli A, Gustafson K, Newman D E and Sánchez R 2014 Transport equation describing fractional lévy motion of suprathermal ions in torpex Nucl. Fusion 54104009

[15] Fasoli A et al 2010 Electrostatic instabilities, turbulence and fast ion interactions in the torpex device Plasma Phys. Control. Fusion $\mathbf{5 2} 124020$

[16] Furno I et al 2015 Plasma turbulence, suprathermal ion dynamics and code validation on the basic plasma physics device torpex J. Plasma Phys. 81345810301

[17] Spolaore M et al 2015 Electromagnetic turbulent structures: a ubiquitous feature of the edge region of toroidal plasma configurations Phys. Plasmas 22012310

[18] Park J, Lühr H, Stolle C, Rother M, Min K W and Michaelis I 2010 Field-aligned current associated with low-latitude plasma blobs as observed by the champ satellite Annal. Geophys. 28 697-703

[19] Avino F, Fasoli A and Furno I 2014 The new torpex in-vessel toroidal conductor for the generation of a poloidal magnetic field Rev. Sci. Instrum. 85033506

[20] Bovet A, Furno I, Fasoli A, Gustafson K and Ricci P 2013 Three-dimensional measurements of non-diffusive fast ion transport in torpex Plasma Phys. Control. Fusion 55124021

[21] Bovet A, Fasoli A, Ricci P, Furno I and Gustafson K 2015 Nondiffusive transport regimes for suprathermal ions in turbulent plasmas Phys. Rev. E 91041101

[22] Furno I et al 2008 Experimental observation of the blobgeneration mechanism from interchange waves in a plasma Phys. Rev. Lett. 100055004

[23] Müller S H et al 2008 Plasma blobs in a basic toroidal experiment: origin, dynamics and induced transport Phys. Plasmas 14110704

[24] Diallo A, Fasoli A, Furno I, Labit B, Podestà M and Theiler C 2008 Dynamics of plasma blobs in a shear flow Phys. Rev. Lett. 101115005

[25] Poli F M, Brunner S, Diallo A, Fasoli A, Furno I, Labit B, Mueller S H, Plyushchev G and Podestà M 2006 Experimental characterization of drift-interchange instabilities in a simple toroidal plasma Phys. Plasmas 13102104

[26] Poli F M et al 2008 Transition from drift to interchange instabilities in an open magnetic field line configuration Phys. Plasmas 15032104

[27] Theiler C et al 2009 Cross-field motion of plasma blobs in an open magnetic field line configuration Phys. Rev. Lett. 103065001

[28] Theiler C, Furno I, Fasoli A, Ricci P, Labit B and Iraji D 2011 Blob motion and control in simple magnetized plasmasa Phys. Plasmas 18055901

[29] Furno I, Spolaore M, Theiler C, Vianello N, Cavazzana R and Fasoli A 2011 Direct two-dimensional measurements of the field-aligned current associated with plasma blobs Phys. Rev. Lett. 106245001

[30] Furno I, Theiler C, Lanon D, Fasoli A, Iraji D, Ricci P, Spolaore M and Vianello N 2011 Blob current structures in torpex plasmas: experimental measurements and numerical simulations Plasma Phys. Control. Fusion 53124016

[31] Furno I, Theiler C, Chabloz V, Fasoli A and Loizu J 2014 Presheath density drop induced by ion-neutral friction along plasma blobs and implications for blob velocities Phys. Plasmas 21012305

[32] Ricci P and Rogers B N 2010 Turbulence phase space in simple magnetized toroidal plasmas Phys. Rev. Lett. 104145001

[33] Ricci P, Theiler C, Fasoli A, Furno I, Gustafson K, Iraji D and Loizu J 2011 Methodology for turbulence code validation: quantification of simulation-experiment agreement and application to the torpex experiment Phys. Plasmas 18032109 
[34] Ricci P, Halpern F D, Jolliet S, Loizu J, Mosetto A, Fasoli A, Furno I and Theiler C 2012 Simulation of plasma turbulence in scrape-off layer conditions: the gbs code, simulation results and code validation Plasma Phys. Control. Fusion $\mathbf{5 4} 124047$

[35] Podestà M, Fasoli A, Labit B, Furno I, Ricci P, Poli F M, Diallo A, Müller S H and Theiler C 2008 Cross-field transport by instabilities and blobs in a magnetized toroidal plasma Phys. Rev. Lett. 101045001

[36] Labit B, Theiler C, Fasoli A, Furno I and Ricci P 2011 Blobinduced toroidal momentum transport in simple magnetized plasmas Phys. Plasmas 18032308

[37] Labit B et al 2007 Statistical properties of electrostatic turbulence in toroidal magnetized plasmas Plasma Phys. Control. Fusion 49 B281

[38] Labit B, Furno I, Fasoli A, Diallo A, Müller S H, Plyushchev G, Podestà M and Poli F M 2007 Universal statistical properties of drift-interchange turbulence in torpex plasmas Phys. Rev. Lett. 98255002

[39] Heidbrink W W et al 2012 Measurements of interactions between waves and energetic ions in basic plasma experiments Plasma Phys. Control. Fusion 54124007

[40] Plyushchev G et al 2006 Fast ion source and detector for investigating the interaction of turbulence with suprathermal ions in a low temperature toroidal plasma Rev. Sci. Instrum. 77 10F503
[41] Bovet A, Furno I, Fasoli A, Gustafson K and Ricci P 2012 Investigation of fast ion transport in torpex Nucl. Fusion 52094017

[42] Ricci P, Rogers B N and Brunner S 2008 High- and lowconfinement modes in simple magnetized toroidal plasmas Phys. Rev. Lett. 100225002

[43] Ricci P and Rogers B N 2009 Transport scaling in interchange-driven toroidal plasmas Phys. Plasmas 16062303

[44] Bovet A, Fasoli A and Furno I 2014 Time-resolved measurements of suprathermal ion transport induced by intermittent plasma blob filaments Phys. Rev. Lett. 113225001

[45] Schreiber T 2000 Measuring information transfer Phys. Rev. Lett. 85 461-4

[46] van Milligen B Ph, Birkenmeier G, Ramisch M, Estrada T, Hidalgo C and Alonso A 2014 Causality detection and turbulence in fusion plasmas Nucl. Fusion 54023011

[47] Müller S H 2006 Probabilistic analysis of turbulent structures from two-dimensional plasma imaging Phys. Plasmas 13100701

[48] Furno I et al 2008 Mechanism for blob generation in the torpex toroidal plasmaa Phys. Plasmas 15055903

[49] Gustafson K and Ricci P 2012 Lévy walk description of suprathermal ion transport Phys. Plasmas 19032304 